\title{
A MULTIPLIER THEOREM FOR FOURIER TRANSFORMS
}

BY

JAMES D. MCCALL, JR.

ABSTRACT. A function $f$ analytic in the upper half-plane $\Pi^{+}$is said to be of class $E_{p}\left(\Pi^{+}\right)(0<p<\infty)$ if there exists a constant $C$ such that $\int_{-\infty}^{\infty}|f(x+i y)|^{\prime} d x \leq C<\infty$ for all $y>0$. These classes are an extension of the $H_{p}$ spaces of the unit disc $U$. For $f$ belonging to $E_{p}\left(\Pi^{+}\right)(0<p \leq 2)$, there exists a Fourier transform $\hat{f}$ with the property that $f(z)=(2 \pi)^{-1} \int_{0}^{\infty} \hat{f}(t) e^{i z t} d t$. This makes it possible to give a definition for the multiplication of $E_{p}\left(\Pi^{+}\right)(0<p \leq 2)$ into $L_{q}(0, \infty)$ that is analogous to the multiplication of $H_{p}(U)$ into $l_{q}$. In this paper, we consider the case $0<p<1$ and $p \leq q$ and derive a necessary and sufficient condition for multiplying $E_{p}\left(\Pi^{+}\right)$into $L_{q}(0, \infty)$.

1. Introduction. A function $f$ analytic in the unit disc $U$ is said to be of class $H_{p}(U)$ if there exists a constant $C$ such that $\int_{0}^{2 \pi}\left|f\left(r e^{i \theta}\right)\right|^{p} d \theta \leq C<\infty$ for all $r<1$. For these classes there exists a rich and varied theory which is described in Duren's book [2]. Among the concepts studied is that of multipliers from $H_{p}(U)$ to $l_{q}$.

Definition 1. A sequence $\left\{\lambda_{n}\right\}$ is said to multiply $H_{p}(U)$ into $l_{q}(0<q<\infty)$, if for each $f(z)=\sum a_{n} z^{n}$ belonging to $H_{p}(U), \sum\left|a_{n}\right|^{q}\left|\lambda_{n}\right|^{q}<\infty$.

Duren and Shields have shown that a necessary and sufficient condition for $\left\{\lambda_{n}\right\}$ to multiply $H_{p}(U)(0<p<1)$ into $l_{q}(p \leq q<\infty)$ is that

$$
\sum_{n=1}^{N} n^{q / p}\left|\lambda_{n}\right|^{q}=O\left(N^{q}\right) \quad[2],[3] \text {. }
$$

It is our aim in this paper to consider classes of functions analytic in the upper half-plane $\Pi^{+}$, which are analogous to the classes $H_{p}(U)$, and to prove a result similar to that of Duren and Shields.

2. The main result.

Definition 2. A function $f$ analytic in $\Pi^{+}$is said to be of class $E_{p}\left(\Pi^{+}\right)$ $(0<p<\infty)$ if there exists a constant $C$ such that

$$
M_{p}(y, f)=\left\{\int_{-\infty}^{\infty}|f(x+i y)|^{p} d x\right\}^{1 / p} \leq C<\infty
$$

for all $0<y<\infty$.

The expression $M_{p}(y, f)$ is called a $p$ th mean of $f$. Also the expression $M_{\infty}(y, f)=\sup _{-\infty<x<\infty}|f(x+i y)|$ is a $p$ th mean of $f$ and, if $M_{\infty}(y, f)$ is bounded, $f$ is said to belong to $E_{\infty}\left(\Pi^{+}\right)$.

Received by the editors July 18, 1972 and, in revised form, December 14, 1972.

AMS (MOS) subject classifications (1970). Primary 30A78; Secondary 42A68.

Key words and phrases. $H_{p}$ spaces, multipliers. 
Definition 3. If $f$ belongs to $E_{p}\left(\Pi^{+}\right)(0<p<1)$, then the Fourier transform of $f$ is

$$
\hat{f}(t)=\int_{-\infty}^{\infty} f(x+i y) e^{-i(x+i y) t} d x \quad[6] .
$$

A proof of the fact that $\hat{f}$ exists and is independent of $y$ is given in $\$ 5$. In addition, the facts $\hat{f}(t)$ is continuous, $\hat{f}(t)=0$ for $t \leq 0$, and

$$
f(z)=(2 \pi)^{-1} \int_{0}^{\infty} \hat{f}(t) e^{i z t} d t
$$

are proved there.

Definition 4. Let $\phi(t)$ be a function measurable on $(0, \infty)$. Then $\phi(t)$ is said to multiply $E_{p}\left(\Pi^{+}\right)(0<p<1)$ into $L_{q}(0, \infty)(0<q<\infty)$, if for each $f(z)$ $=(2 \pi)^{-1} \int_{0}^{\infty} \hat{f}(t) e^{i z t} d t$ belonging to $E_{p}\left(\Pi^{+}\right)$,

$$
\int_{0}^{\infty}|\phi(t)|^{q}|\hat{f}(t)|^{q} d t<\infty
$$

We now state the main result.

Theorem A. Let $\phi(t)$ be a function measurable on $(0, \infty)$. Then $\phi(t)$ multiplies $E_{p}\left(\Pi^{+}\right)$into $L_{q}(0, \infty)(p \leq q)$ if and only if

$$
\int_{0}^{X}{ }^{q / p}|\phi(t)|^{X} d t \leq K X^{q}
$$

where $K$ is a positive constant.

The proof of Theorem A requires the use of two other results.

Theorem B. If $0<p<q \leq \infty$, $f$ belongs to $E_{p}\left(\Pi^{+}\right), \alpha=1 / p-1 / q$, and $\lambda \geq p$, then $\int_{0}^{\infty} y^{\lambda \alpha-1} M_{q}^{\lambda}(y, f) d y<\infty$.

The second of these results needs some introduction. If $f$ belongs to $E_{p}\left(\Pi^{+}\right)$ $(0<p<\infty)$, then $\lim _{y \rightarrow 0} f(x+i y)=f(x)$ exists a.e. and

$$
\rho(f, g)=\int_{0}^{\infty}|f(x)-g(x)|^{p} d x,
$$

where $f$ and $g$ belong to $E_{p}\left(\Pi^{+}\right)$, is a translation invariant metric on $E_{p}\left(\Pi^{+}\right)$. Moreover, under this metric, $E_{p}\left(\Pi^{+}\right)(0<p<\infty)$ is a complete topological vector space. In other words, $E_{p}\left(\Pi^{+}\right)(0<p<\infty)$ is an $F$-space [1], [2], [5]. Finally, we say that an operator $\Lambda$ from $E_{p}\left(\Pi^{+}\right)$into $L_{q}(0, \infty)$ is bounded if there exists a constant $K$ such that $\|\Lambda(f)\|_{q}<K\|f\|_{p}$, where $\|f\|_{p}=\left\{\int_{0}^{\infty}|f(x)|^{p} d x\right\}^{1 / p}$.

Theorem C. Let $\phi(t)$ be a function measurable on $(0, \infty)$. If $\phi(t)$ multiplies $E_{p}\left(\Pi^{+}\right)(0<p<1)$ into $L_{q}(0, \infty)$ then the operator $\Lambda(f)(t)=\phi(t) \hat{f}(t)$ is bounded. 
We defer, for now, the proofs of Theorem B and Theorem $\mathbf{C}$ in order to give an immediate proof of Theorem $A$.

Proof of Theorem A. We begin by showing that (1) is necessary. So let us consider the function

$$
F(z)=F_{\rho}(z)=(2 \pi)^{-1} \int_{0}^{\infty} t^{1 / p} e^{-\rho t} e^{i t z} d t .
$$

Since the Laplace transform of $t^{u-1}(u>0)$ is $\Gamma(u) / s^{u}$, where $s$ is a complex number with $\operatorname{Re} s>0$, we see that setting $u=1+1 / p$ and $s=-i z+\rho$ gives

$$
F(z)=\Gamma(1+1 / p) /(\rho-i z)^{1+1 / p}
$$

From this it follows that $F(z)$ belongs to $E_{p}\left(\Pi^{+}\right)$and $\|F\|_{p}=M / \rho$. But by Theorem $\mathrm{C}$ there exists a constant $K$ such that

$$
\|\Lambda(f)\|_{q} \leq K\|F\|_{p}
$$

so $\|\hat{F}(t) \phi(t)\|_{q} \leq K M / \rho$. Thus, our next step is to find $\hat{F}(t)$. However, $F(x+i y)$ $=F_{y}(x)$ is in $L_{1}(-\infty, \infty)$ and is the Fourier transform of

$$
\begin{aligned}
g(t) & =(2 \pi)^{-1} t^{1 / p} e^{-p t} e^{-y t} & & \text { if } t \geq 0, \\
& =0 & & \text { if } t<0,
\end{aligned}
$$

which also belongs to $L_{1}(-\infty, \infty)$. Hence $\hat{F}(t) e^{-y t}=\hat{F}_{y}(t)=2 \pi g(t)$ or $\hat{F}(t)$ $=t^{1 / p} e^{-\rho t}$ if $t \geq 0$ and zero if $t<0$ [7]. Consequently,

$$
\int_{0}^{\infty} t^{q / p}|\phi(t)|^{q} e^{-q \rho t} d t \leq K^{q} M^{q} / \rho^{q}
$$

and this implies that

$$
\int_{0}^{X} e^{-q \rho X} t^{q / p}|\phi(t)|^{q} d t \leq K^{q} M^{q} / \rho^{q}
$$

for $X>0$. So taking $\rho=1 / X$, we find

$$
\int_{0}^{X}{ }^{q}{ }^{q / p}|\phi(t)|^{q} d t \leq K M^{q} e^{q} X^{q} .
$$

To prove that (1) is sufficient, we begin by considering the integral

$$
\int_{0}^{\infty} t^{q / p}|\phi(t)|^{q} e^{-y t} d t \quad(y>0) .
$$

Letting $S(t)=\int_{0}^{t} \tau^{q / p}|\phi(\tau)|^{q} d t$ and integrating by parts we find that when we use the estimate $S(t) \leq K t^{q}$, the integral is less than or equal to $K y \int_{0}^{\infty} t^{q} e^{-y t} d t$ $=K \Gamma(q+1) / y^{q}$. Hence

$$
y^{q} \int_{0}^{\infty}{ }^{q / p}|\phi(t)|^{q} e^{-y t} d t \leq C<\infty
$$


for $y>0$. Next we note that for $\gamma=q(1 / p-1)$, Theorem B implies that for $f$ belonging to $E_{p}\left(\Pi^{+}\right)(0<p<1)$,

$$
\int_{0}^{\infty} y^{\gamma-1} M_{1}^{q}(y, f) d y<\infty .
$$

Thus for each $f$ belonging to $E_{p}\left(\Pi^{+}\right)$

$$
\int_{0}^{\infty} y^{\gamma-1} M_{1}^{q}(y, f)\left[y^{q} \int_{0}^{\infty} t^{q / p}|\phi(t)|^{q} e^{-y t} d t\right] d y<\infty,
$$

or using Fubini's theorem

$$
\int_{0}^{\infty} \int_{0}^{\infty} t^{q / p}|\phi(t)|^{q} y^{\gamma+q-1} M_{1}^{q}(y, f) e^{-y t} d y d t<\infty .
$$

But from the definition of the Fourier transform for $f$, we have $|\hat{f}(t)| e^{-y t}$ $\leq M_{1}(y, f)$. Thus

$$
\int_{0}^{\infty}|\phi(t)|^{q}|\hat{f}(t)|^{q} t^{q / p} \int_{0}^{\infty} y^{\gamma+q-1} e^{-(q+1) y t} d y d t<\infty
$$

or

$$
\frac{\Gamma(q / p)}{(q+1)^{q / p}} \int_{0}^{\infty}|\phi(t)|^{q}|\hat{f}(t)|^{q} d t<\infty .
$$

Theorem A has the following interesting corollary.

Corollary. If $f$ belongs to $E_{p}\left(\Pi^{+}\right)(0<p<1)$, then $\int_{0}^{\infty}|\hat{f}(t)|^{p} t^{p-2} d t<\infty$.

This is an extension of the following results.

Theorem (Hardy-Littlewood-Titchmarsh). If $f$ belongs to $E_{p}\left(\Pi^{+}\right)(1<p \leq 2)$, then $\int_{0}^{\infty}|\hat{f}(t)|^{p} t^{p-2} d t<\infty[8]$.

Theorem (Hille-Tamarkin). If $f$ belongs to $E_{1}\left(\Pi^{+}\right)$, then $\int_{0}^{\infty}|\hat{f}(t)| / t d t<\infty[4]$.

3. The proof of Theorem B. This proof is a consequence of several other theorems.

Theorem 1. Let $u(z)$ be a nonnegative subharmonic function defined on $\Pi^{+}$and suppose

$$
\int_{-\infty}^{\infty} u(x+i y) d x \leq C / y^{\alpha} \quad(y>0),
$$

where $\alpha \geq 0$. Then there exists a constant $K=K(\alpha)$ such that $u\left(x_{0}+i y_{0}\right)$ $\leq K C / y_{0}^{\alpha+1}$ for each point $z_{0}=x_{0}+i y_{0}\left(y_{0}>0\right)$.

Proof. The case $\alpha=0$ was proved by Krylov [5]. So assume $\alpha>0$. Then setting $y_{1}=y_{0} / 2$ and $u_{y_{1}}(z)=u\left(x+i\left(y+y_{1}\right)\right)$, we find

$$
\int_{-\infty}^{\infty} u_{y_{1}}(x+i y) d x \leq C / y_{1}^{\alpha} \quad(y>0) \text {. }
$$


Hence, by the case $\alpha=0$, we have $u_{y_{1}}\left(x_{0}+i y_{2}\right) \leq K C / y_{1}^{\alpha} y_{2}\left(y_{2}>0\right)$, and putting $y_{1}=y_{2}=y_{0} / 2$,

$$
u\left(x_{0}+i y_{0}\right) \leq 2^{\alpha+1} K C / y_{0}^{\alpha+1} .
$$

Theorem 2. Suppose $f(z)$ is analytic in $\Pi^{+}$and

$$
M_{p}(y, f) \leq C / y^{\beta} \quad(0<p<\infty, \beta \geq 0) .
$$

Then there exists a constant $K=K(\beta, p, q)$ such that

$$
M_{q}(y, f) \leq K C / y^{\beta+1 / p-1 / q} \quad(p<q \leq \infty) .
$$

Proof. It suffices to consider the case $q=\infty$. For suppose (2) has been proven for $q=\infty$ and $K \geq 1$ (which we may assume without loss of generality). Then

$$
\begin{aligned}
M_{q}(y, f) & =\left\{\int_{\infty}^{\infty}|f(x+i y)|^{p}|f(x+i y)|^{q-p} d x\right\}^{1 / q} \\
& \leq\left[M_{\infty}(y, f)\right]^{q-p / q}\left[M_{p}(y, f)\right]^{p / q} \\
& \leq K^{q-p / q} C / y^{\lambda}
\end{aligned}
$$

where $\lambda=\beta+1 / p-1 / q$. Now to derive the theorem for $q=\infty$, let $u(z)$ be the nonnegative subharmonic function $|f(z)|^{p}$ and $\alpha=\beta p$. Then Theorem 1 implies

$$
\left|f\left(x_{0}+i y_{0}\right)\right|^{p} \leq K C / y_{0}^{\beta p+1},
$$

which is equivalent to (2).

Theorem 3. Suppose $f$ belongs to $E_{p}\left(\Pi^{+}\right)$. Then for $1<p<\infty,-1<b$, and $1<a<\infty$,

$$
\int_{0}^{\infty} y^{b} M_{p}^{a}(y, f) d y \leq C \int_{0}^{\infty} y^{a+b} M_{p}^{a}\left(y, f^{\prime}\right) d y,
$$

where $C=C(a, b)$ is independent of $f$.

Proof. We begin by assuming that $f$ is analytic in the closed upper half-plane. Then integrating by parts we find

$$
\begin{aligned}
\int_{0}^{y_{0}} y^{b} M_{p}^{a}(y, f) d y= & \frac{y^{b+1}}{b+1} M_{p}^{a}\left(y_{0}, f\right) \\
& -\frac{1}{b+1} \int_{0}^{y_{0}} y^{b+1} \frac{\partial}{\partial y}\left\{M_{p}^{a}(y, f)\right\} d y .
\end{aligned}
$$

Thus our next step is to estimate $\left|(\partial / \partial y) M_{p}^{a}(y, f)\right|$. But

$$
(\partial / \partial y) M_{p}^{a}(y, f)=(a / p) M_{p}^{a-p}(y, f)(\partial / \partial y) M_{p}^{p}(y, f),
$$


so we need to estimate $\left|(\partial / \partial y) M_{p}^{P}(y, f)\right|$.

However,

$$
\left.\left.\left|\frac{\partial}{\partial y}\right| f(x+i y)\right|^{p}|=p| f(x+i y)\right|^{p-1}\left|\frac{\partial}{\partial y}\right| f(x+i y)||
$$

and

$$
\frac{\| f\left(x+i y_{1}\right)|-| f\left(x+i y_{2}\right)||}{\left|y_{1}-y_{2}\right|} \leq \frac{\left|f\left(x+i y_{1}\right)-f\left(x+i y_{2}\right)\right|}{\left|y_{1}-y_{2}\right|}
$$

implies

$$
|(\partial / \partial y)| f(x+i y)|| \leq\left|f^{\prime}(x+i y)\right|
$$

so

$$
\left.\left.\left|\frac{\partial}{\partial y}\right| f(x+i y)\right|^{p}|\leq p| f(x+i y)\right|^{p-1}\left|f^{\prime}(x+i y)\right|
$$

Thus Hölder's inequality implies

$$
\left|(\partial / \partial y) M_{p}^{p}(y, f)\right| \leq p M_{p}^{p-1}(y, f) M_{p}\left(y, f^{\prime}\right)
$$

and this implies

$$
\left|(\partial / \partial y) M_{p}^{a}(y, f)\right| \leq a M_{p}^{a-1}(y, f) M_{p}\left(y, f^{\prime}\right) .
$$

But now we have

$$
\begin{aligned}
\left|\int_{0}^{y_{0}} y^{b+1} \frac{\partial}{\partial y}\left\{M_{p}^{a}(y, f)\right\} d y\right| & \leq a \int_{0}^{y_{0}} y^{b+1} M_{p}^{a-1}(y, f) M_{p}\left(y, f^{\prime}\right) d y \\
\leq & a\left\{\int_{0}^{y_{0}} y^{b} M_{p}^{a}(y, f) d y\right\}^{1-1 / a}\left\{\int_{0}^{y_{0}} y^{a+b} M_{p}^{a}\left(y, f^{\prime}\right) d y\right\}^{1 / a},
\end{aligned}
$$

where we have used Hö!der's inequality again. Hence

$$
\begin{aligned}
& \left\{\int_{0}^{y_{0}} y^{b} M_{p}^{a}(y, f) d y\right\}^{1 / a} \\
& \quad \leq\left(\frac{y_{0}^{b+1}}{b+1}\right)^{1 / a} M_{p}\left(y_{0}, f\right)+\frac{a}{b+1}\left\{\int_{0}^{y_{0}} y^{a+b} M_{p}^{a}\left(y, f^{\prime}\right) d y\right\}^{1 / a} .
\end{aligned}
$$

where we have used the estimate

$$
\int_{0}^{y_{0}} y^{b} M_{p}^{a}(y, f) d y \geq \frac{y_{0}^{b+1} M_{p}^{a}\left(y_{0}, f\right)}{b+1} .
$$


which follows from the fact that the means $M_{p}(y, f)$ are nonincreasing functions of $y[5]$.

From (4), it is clear that in order to complete the proof for this case, we need only show that $y_{0}^{b+1} M_{p}^{a}\left(y_{0}, f\right)$ tends to zero as $y_{0}$ tends to infinity. But using Theorem 2, it is easy to see that $f\left(x+i y_{0}\right)=-i \int_{y_{0}}^{\infty} f^{\prime}(x+i y) d y$ and applying Minkowski's inequality, we find

$$
M_{p}\left(y_{0}, f\right) \leq \int_{y_{0}}^{\infty} M_{p}\left(y, f^{\prime}\right) d y .
$$

So suppose $r>1$. Then

$$
M_{p}^{a}\left(y_{0}, f\right) \leq\left[C\left(y_{0}\right)\right]^{a}\left[\frac{1}{r-1} \int_{y_{0}}^{\infty} y^{r} M_{p}\left(y, f^{\prime}\right) \frac{d\left(-1 / y^{r-1}\right)}{C\left(y_{0}\right)}\right]^{a},
$$

where $C\left(y_{0}\right)=\int_{y_{0}}^{\infty} d\left(-1 / y^{r-1}\right)=1 / y_{0}^{r-1}$, and Jensen's inequality gives

$$
M_{p}^{a}\left(y_{0}, f\right) \leq\left[C\left(y_{0}\right)\right]^{a-1} \frac{1}{(r-1)^{a-1}} \int_{y_{0}}^{\infty} y^{a r-r} M_{p}^{a}\left(y, f^{\prime}\right) d y .
$$

Hence setting $r=(a+b) /(a-1)$, we have

$$
y_{0}^{b+1} M_{p}^{a}\left(y_{0}, f\right) \leq \frac{1}{((b+1) /(a-1))^{a-1}} \int_{y_{0}}^{\infty} y^{a+b} M_{p}^{a}\left(y, f^{\prime}\right) d y,
$$

from which it follows that $y_{0}^{b+1} M_{p}^{a}\left(y_{0}, f\right)$ tends to zero as $y_{0}$ tends to infinity.

Finally we remove the restriction that $f$ is analytic in the closed upper halfplane. Since $f_{y}(z)=f(z+i y)$ is analytic in the closed upper half-plane, the theorem holds for $f_{y}(z)$. Thus the result for $f(z)$ follows from letting $y$ tend to zero and applying the monotone convergence theorem.

These three theorems have prepared the way for a proof of Theorem B.

Proof of Theorem B. We first reduce the theorem to the case $\lambda=p=2$. By Theorem 2

$$
M_{p}^{\lambda}(y, f) \leq K^{\lambda-p} M_{q}^{p}(y, f) / y^{\alpha(\lambda-p)},
$$

so

$$
\int_{0}^{\infty} y^{\alpha \lambda-1} M_{q}^{\lambda}(y, f) d y \leq K^{\lambda-p} \int_{0}^{\infty} y^{\alpha p-1} M_{q}^{p}(y, f) d y .
$$

Hence we can assume $\lambda=p$. Next assume the theorem is true for $\lambda=p=2$ and $f(z) \neq 0$ in $\Pi^{+}$and belongs to $E_{p}\left(\Pi^{+}\right)$. Then $g(z)=[f(z)]^{p / 2}$ belongs to $E_{2}\left(\Pi^{+}\right)$and

$$
\int_{0}^{\infty} y^{-p / q} M_{q}^{p}(y, f) d y=\int_{0}^{\infty} y^{-2 / s} M_{s}^{2}(y, g) d y<\infty
$$


where $s=2 q / p>2$. In case $f(z)$ has zeros in $\Pi^{+}$, it is possible to write it as a sum of two nonzero functions in $E_{p}\left(\Pi^{+}\right)[2]$ and still show that it suffices to take $p=2$.

So let $f \in E_{2}\left(\Pi^{+}\right)$. Then using the Paley-Wiener theorem [7], we can write

$$
f(z)=\frac{1}{2 \pi} \int_{0}^{\infty} \hat{f}(t) e^{i z t} d t,
$$

where $\hat{f}(t)$ is the Fourier transform of the boundary function $f(x)$ of $f(z)$. Also

$$
f^{\prime}(z)=\frac{1}{2 \pi} \int_{0}^{\infty} t \hat{f}(t) e^{i z t} d t .
$$

Next we assume $2<q<\infty$. Then by Theorem 3

$$
\int_{0}^{\infty} y^{-2 / q} M_{q}^{2}(y, f) d y \leq C \int_{0}^{\infty} y^{2-2 / q} M_{q}^{2}\left(y, f^{\prime}\right) d y,
$$

and by Theorem $2 M_{q}\left(y, f^{\prime}\right) \leq K y^{1 / q-1 / 2} M_{2}\left(y / 2, f^{\prime}\right)$, so

$$
\int_{0}^{\infty} y^{-2 / q} M_{q}^{2}(y, f) d y \leq C K \int_{0}^{\infty} y M_{2}^{2}\left(y / 2, f^{\prime}\right) d y .
$$

Finally, by Plancherel's theorem [7], we find

$$
\begin{aligned}
\int_{0}^{\infty} y^{-2 / q} M_{q}^{2}(y, f) d y & \leq \frac{C K}{2 \pi} \int_{0}^{\infty} y \int_{0}^{\infty}|\hat{f}(t)|^{2} t^{2} e^{-y t} d t d y \\
& =\frac{C K}{2 \pi} \int_{0}^{\infty}|\hat{f}(t)| t^{2} \int_{0}^{\infty} y e^{-y t} d y d t \\
& =\frac{C K}{2 \pi} \int_{0}^{\infty}|\hat{f}(t)|^{2} d t \\
& =C K \int_{0}^{\infty}|f(x)|^{2} d x<\infty
\end{aligned}
$$

If $q=\infty$, then the estimate

$$
M_{\infty}^{2}(y, f) \leq K M_{r}^{2}(y / 2, f) / y^{2 / r}
$$

for some $r>2$ can be used to derive the desired results.

4. The proof of Theorem C. Since $E_{p}\left(\Pi^{+}\right)$is an $F$-space under the metric $\rho(f, g)=\int_{-\infty}^{\infty}|f(x)-g(x)|^{p} d x$, we can use the closed graph theorem. Thus we need to show that $\Lambda$ is a closed operator. So let $\left\{f_{n}\right\}$ be a sequence which converges in $E_{p}\left(\Pi^{+}\right)$to $f$ and also suppose $\Lambda\left(f_{n}\right)(t)=\phi(t) \hat{f}_{n}(t)$ converges to $g(t)$ in $L_{q}(0, \infty)$. Then we need to show that $\Lambda(f)(t)=g(t)$ a.e.

Considering the sequence $\left\{f_{n}\right\}$ and $f$ first, we find by Theorem 2 that

$$
\left\{\int_{-\infty}^{\infty}\left|f_{n}\left(x+i y_{0}\right)-f\left(x+i y_{0}\right)\right|^{2} d x\right\}^{1 / 2} \leq \frac{K\left\|f_{n}-f\right\|_{p}}{y_{0}^{1 / p-1 / 2}}
$$


where $y_{0}>0$. Thus $f_{y_{0}, n}(z)=f_{n}\left(z+i y_{0}\right)$ converges to $f_{y_{0}}(z)=f\left(z+i y_{0}\right)$ in $E_{2}\left(\Pi^{+}\right)$. Moreover, it is easy to see that the Fourier transform of $f_{n, y}(x)$ is $\hat{f}_{n}(t) e^{-y_{0} t}$, while the Fourier transform of $f_{y_{0}}(x)$ is $f(t) e^{-y_{0} t}$. Consequently, Plancherel's theorem [7] implies that $\hat{f}_{n}(t) e^{-y_{0} t}$ converges to $\hat{f}^{\prime}(t) e^{-y_{0} t}$ in $L_{2}(0, \infty)$. Hence, there exists a subsequence $\left\{\hat{f}_{k}(t)\right\}$ of $\left\{\hat{f}_{n}(t)\right\}$ converging to $\hat{f}(t)$ a.e. But the sequence $\left\{\Lambda\left(f_{k}\right)\right\}$ also converges to $g(t)$ in $L_{q}(0, \infty)$. Therefore, there exists a subsequence of $\left\{\Lambda\left(f_{k}\right)\right\}$, which we also denote by $\left\{\Lambda\left(f_{k}\right)\right\}$, converging to $g(t)$ a.e. Thus $\left\{\phi(t) f_{k}(t)\right\}$ converges to $\left.\phi(t)\right\}(t)$ a.e. and also to $g(t)$ a.e., which implies

$$
\phi(t) \hat{f}(t)=g(t) \text { a.e. }
$$

5. Fourier transform. The Fourier transform defined in $\$ 2$ certainly exists since Theorem 2 implies that $f_{y}(x)=f(x+i y)$ belongs to $L_{1}(-\infty, \infty)$. In fact, if $C$ is a constant such that $M_{p}(y, f) \leq C$ for $y>0$, then there exists a constant $K=K(0, p, 1)$ such that

$$
\int_{-\infty}^{\infty}|f(x+i y)| d x \leq C K / y^{1 / p-1}
$$

for $y>0$.

To see that $\hat{f}$ is independent of $y$, fix $0<y_{1}<y_{2}<\infty$ and for each $\alpha>0$ let $\Gamma_{\alpha}$ be the rectangular contour with vertices $\pm \alpha+i y_{1}$ and $\pm \alpha+i y_{2}$. By Cauchy's theorem

$$
\int_{\Gamma_{a}} f(z) e^{-i t z} d z=0 .
$$

Next let $I=\left[y_{1}, y_{2}\right]$ and put

$$
\Phi(\beta)=i \int_{I} f(\beta+i u) e^{-i t \beta} e^{i u} d u .
$$

Then $|\Phi(\beta)| \leq e^{t y_{2}} \int_{y_{1}}^{y_{2}}|f(\beta+i u)| d u$. Now if we let

$$
\Psi(\beta)=\int_{y_{1}}^{y_{2}}|f(\beta+i u)| d u,
$$

then Fubini's theorem and (1) imply

$$
\int_{-\infty}^{\infty} \Psi(\beta) d \beta=\int_{y_{1}}^{y_{2}} \int_{-\infty}^{\infty}|f(\beta+i y)| d \beta d y \leq \frac{C K}{y_{1}^{1 / p-1}}\left(y_{2}-y_{1}\right) .
$$

Thus there exists a sequence $\left\{\alpha_{j}\right\}$ such that $\alpha_{j} \rightarrow \infty$ as $j \rightarrow \infty$ and $\Psi\left(\alpha_{j}\right)$ $+\Psi\left(-\alpha_{j}\right) \rightarrow 0$ as $j \rightarrow \infty$. Hence we have

$$
\Phi\left(\alpha_{j}\right) \rightarrow 0 \text { and } \Phi\left(-\alpha_{j}\right) \rightarrow 0
$$

as $j \rightarrow \infty$. Now combining (1), (2), and (3), we find

$$
\begin{aligned}
\int_{-\infty}^{\infty} f( & \left.x+i y_{1}\right) e^{-i\left(x+i y_{1}\right) t} d x \\
& =\int_{-\infty}^{\infty} f\left(x+i y_{2}\right) e^{-i\left(x+i y_{2}\right) t} d x,
\end{aligned}
$$

i.e., $\hat{f}$ is independent of $y$. 
If we let $f_{y}(z)=f(z+i y)$, then (4) becomes

$$
\hat{f}(t)=e^{y_{1}} \hat{f}_{y_{1}}(t)=e^{y_{2}} \hat{f}_{y_{2}}(t) .
$$

Since $\hat{f}_{y}$ is the Fourier transform of an $L_{1}(-\infty, \infty)$ function, it is continuous and hence $\hat{f}$ is continuous.

Using (1) again, we see that

$$
|\hat{f}(t)| e^{-t y}=\left|\hat{f}_{y}(t)\right| \leq\left\|f_{y}\right\|_{1} \leq C K / y_{0}^{1 / p-1}
$$

for a fixed $y_{0}<y$. Thus if we fix $t<0$ and let $y \rightarrow \infty$, we find $\hat{f}(t)=0$. Hence $\hat{f}(t)$ is identically zero on $(0, \infty)$ and by continuity it is zero at $t=0$. Also note $f_{y}(t) \equiv 0$ on $(-\infty, 0]$.

As we have noted, $\hat{f}(t)=f_{y}(t) e^{y t}$, so $\hat{f}_{y}(t)=\hat{f}(t) e^{-y t}=\hat{f}_{y_{0}}(t) e^{\left(y_{0}-y\right) t}$, and letting $y_{0}=y / 2$, we have

$$
\begin{aligned}
\int_{0}^{\infty}\left|\hat{f}_{y}(t)\right| d t & \leq\left\|f_{y_{0}}\right\|_{1} \int_{0}^{\infty} e^{\left(y_{0}-y\right) t} d t \\
& \leq \frac{K C}{y_{0}^{1 / p-1}} \frac{1}{y-y_{0}} \\
& =\frac{2^{1 / p} K C}{y^{1 / p}} .
\end{aligned}
$$

Hence for $y>0, \hat{f}_{y}$ belongs to $L_{1}(-\infty, \infty)$ and we can apply the inversion theorem [7], to find

$$
\begin{aligned}
f(z) & =f_{y}(x)=(2 \pi)^{-1} \int_{0}^{\infty} \hat{f}_{y}(t) e^{i t x} d t \\
& =(2 \pi)^{-1} \int_{0}^{\infty} \hat{f}(t) e^{-t y} e^{i t x} d t \\
& =(2 \pi)^{-1} \int_{0}^{\infty} \hat{f}(t) e^{i t z} d t .
\end{aligned}
$$

\section{REFERENCES}

1. N. Dunford and J. T. Schwartz, Linear operators. I: General theory, Pure and Appl. Math., vol. 7, Interscience, New York, 1958. MR 22 \# 8302.

2. P. L. Duren, Theory of $H^{P}$ spaces, Pure and Appl. Math., vol. 38, Academic Press, New York, 1970. MR 42 \#3552.

3. P. L. Duren and A. L. Shields, Coefficient multipliers of $H^{p}$ and $B^{p}$ spaces, Pacific J. Math. 32 (1970), 69-78. MR 41 \# 485.

4. E. Hille and J. D. Tamarkin, On the absolute integrability of Fourier transforms, Fund. Math. 25 (1935), 329-352.

5. V. I. Krylov, On functions regular in a half-plane, Mat. Sb. 6 (48) (1939), 95-138; English transl,, Amer. Math. Soc. Transl. (2) 32 (1963), 37-81. MR 1, 308. 
6. E. Stein, Classes $H^{p}$, multiplicateurs et fonctions de Littlewood-Paley, C. R. Acad. Sci. Paris Sér. A-B 263 (1966), A716-A719; A780-A781. MR 37 \#695a,b.

7. W. Rudin, Real and complex analysis, McGraw-Hill, New York, 1966. MR 35 \#1420.

8. E. C. Titchmarsh, Introduction to the theory of Fourier integrals, 2nd ed., Oxford Univ. Press, London, 1948.

Department of Mathematics, Le Moyne-Owen College, Memphis, Tennessee 38126 